\title{
Review of: "The key features of SARS-CoV-2 leader and NSP1 required for viral escape of NSP1-mediated repression"
}

\author{
Mihaela lleana Ionescu ${ }^{1}$ \\ 1 University of Medicine and Pharmacy of Cluj-Napoca
}

Potential competing interests: The author(s) declared that no potential competing interests exist.

The manuscript is focused on a subject of major interest - the elucidation of viral escape and the role of NSP1. I would like to make some comments.

In order to clarify the functions of the SARS-CoV-2 NSP 1, Bujanic L. et al. used " a combination of reporter assays, mutagenesis and mass spectrometry". For the clarity, the lecturer would expect to read about the "combination of reporter assays" used.

In my opinion, the last paragraph of the Introduction section should be re-phrased. The aim of the study should be distinct from the outcome of the study. However I would like to make some comments. The authors should specify which bat coronavirus show "96\% identity at the genome level with SARS-CoV$2 "$ and what method was used to analyze gene homology.

For clarity which IFN? "preventing full induction of interferon (IFN)"

The results of the study add valuable results about the function of SARS-CoV-2 NSP1. However, in my opinion it is too optimistic to conclude "Stem-loop 1 (SL1) is both necessary and sufficient for NSP1mediated upregulation of viral RNA expression". Also, would be useful if the authors explained the selection of the methods included in the study - advantages and the limits of the tests. The authors themselves conclude "An intriguing question remains regarding the extent to which NSP1 upregulates the expression of viral RNAs in the context of actual viral infection." Only "Future in vivo experiments" could advance the understanding of the NSP1-mediated upregulation of viral RNA expression? In my opinion, the results are too speculative and need a deeper analysis.

"NSP1's fundamental role in viral infections make it a highly interesting potential target for drugs." Please, be more specific about the drugs that target NSP1.

"On the SL1 side, finding compounds that target three crucial cytosines (C15, C19 and C20), alone or in combination, might hold a great potential for the development of novel SARS-CoV-2 therapies. "The same, is is obvious that finding a specific drug against SAR-CoV-2 infection is of great interest. But, in my opinion, the authors should provide some clues about the nature of the drugs/inhibitors 
that could be studied in perspective. I

Minor comments:

- all the acronyms should be explained - e.g. HEK293T, STAT1, IAA, RT, FT etc.

- check the manuscript for lack of clarity - e.g. DNA construct paragratesph - "Mutations of SL1, indicated in the figures, were ..." specify the figure.

- add the references for all the tools used (e.g. BiolD)

- I am not qualified to judge the English writing, but some sentences could be re-phrased for clarity - e.g. " Here, too, attempts to resolve the underlying mechanisms have produced conflicting results. As with studies ..."

In my opinion, the research of Bujanic L. et al. is of great interest because the elucidation of the regulation of RNA expression greatly advances the understanding of the SARS-CoV-2 infection. 